\title{
LEGITIMAR PSIQUIATRAS ANTES QUE CURAR PACIENTES. LAS TERAPIAS DE SHOCK EN BUENOS AIRES, ARGENTINA (1930-1970)
}

\author{
Alejandra Golcman \\ CONICET-Universidad Nacional de Tucumán \\ Email: alejandragolcman@gmail.com \\ ORCID iD: http://orcid.org /0000-0002-6609-5749
}

Recibido: 25 noviembre 2015; aprobado: 30 septiembre 2016.

\begin{abstract}
Cómo citar este artículo/Citation: Golcman, Alejandra (2017), “Legitimar psiquiatras antes que curar pacientes. Las terapias de shock en Buenos Aires, Argentina (1930-1970)", Asclepio, 69 (1): p176. doi: http://dx.doi.org/10.3989/asclepio.2017.08
\end{abstract}

RESUMEN: Esta investigación pretende explicar el uso teórico y clínico que se hizo de las terapias de shock en el campo psiquiátrico argentino, particularmente en la provincia de Buenos Aires entre las décadas de 1930 y 1970.

Se sostiene que es preciso estudiar el trabajo psiquiátrico teórico y clínico para conocer cómo los psiquiatras interpretaron la patología mental en el encuentro con sus pacientes. De este modo se analizan dos escenarios bien diferenciados: por un lado, el conformado por el ámbito académico, haciendo hincapié en textos médicos que plasmaron debates que tuvieron lugar en congresos, jornadas, cátedras universitarias, y por otro lado en ámbitos hospitalarios, representado por una población de pacientes crónicas, en un hospital de las afueras de la Capital Federal, el Hospital Esteves de Lomas de Zamora.

El uso de las terapias de shock en manos de médicos psiquiatras, modificó el modo de entender la locura y transformó el derrotero cotidiano de las instituciones hospitalarias. Por lo tanto, la presencia de dichas terapias significó un hecho relevante para legitimar la disciplina, independientemente de la "eficacia" de las mismas en el tratamiento concreto con pacientes.

PALABRAS CLAVE: Terapias de Shock; Prácticas Psiquiátricas; Textos Médicos; Legitimación Disciplinar; Argentina.

\section{LEGITIMING PSYCHIATRISTS RATHER THAN CURING PATIENTS. THE SHOCK THERAPY IN BUENOS AIRES, ARGENTINA (1930-1970)}

ABSTRACT: This research aims to explain the theoretical and clinical use of shock therapies in Argentine psychiatric field, particularly in the province of Buenos Aires between 1930 and 1970.

It is argued that it is necessary to study the theoretical and clinical psychiatric work, in order to learn how psychiatrists interpreted mental pathology. Thus two distinct scenarios are analyzed: first academia, emphasizing on medical texts expressing discussions held at congresses, conferences, university chairs, and secondly in hospital settings, represented by a population of chronic patients in a hospital on the outskirts of the Capital Federal, the Esteves Hospital of Lomas de Zamora.

The use of shock therapy in the hands of psychiatrists, changed the way of understanding madness and transformed the daily course of hospitals. Therefore, the presence of such therapies was relevant to legitimize the discipline, regardless of the "effectiveness" with patients.

KEY WORDS: Shock Therapies; Psychiatric Practices; Medical Texts; Disciplinary Legitimation; Argentina.

Copyright: (C) 2017 CSIC. Este es un artículo de acceso abierto distribuido bajo los términos de la licencia Creative Commons Attribution (CC BY) España 3.0. 


\section{INTRODUCCIÓN}

El uso de las terapias de shock por parte de médicos psiquiatras modificó el modo de entender la locura y la manera de proceder ante ella a nivel mundial. Asimismo, esta práctica transformó el derrotero cotidiano de las instituciones hospitalarias, al brindar una nueva herramienta para "contener" y organizar a sus poblaciones. Como es sabido, con el uso de ciertas terapias de choque primero, y de medicamentos desde la década del cincuenta, la práctica psiquiátrica comenzó a equipararse con las restantes especialidades médicas. Por lo tanto, su utilización significó un hecho relevante para la disciplina, independientemente de su eficacia en el tratamiento concreto brindado a los pacientes. Es en este sentido que esta investigación pretende explicar el uso teórico y clínico que se hizo de las terapias de shock en el campo psiquiátrico argentino, particularmente en la provincia de Buenos Aires entre las décadas de 1930 y 1970.

En este artículo se pone la lupa en textos médicos publicados, como dispositivo para comprender las ideas de los psiquiatras sobre dichos tratamientos y su interpretación de las lecturas extranjeras sobre la temática; es decir, en los debates que tuvieron lugar a lo largo de distintos congresos, jornadas, cátedras universitarias y en ámbitos hospitalarios, y que se plasmaron en dichos artículos. Luego de una lectura exhaustiva, se seleccionaron algunos trabajos para destacar aquellos autores que lograron sistematizar ideas, mostraron cuál sería la aplicación ideal y la posible de estas terapéuticas, o que se posicionaron con algún argumento fuerte al respecto. En el ámbito clínico, se toma para esta investigación una población de pacientes crónicas, en un hospital de las afueras de la Capital Federal. Específicamente, se pretende explicar el uso de terapias de shock, cómo se utilizaron las nuevas tecnologías que llegaban a la institución y qué vinculaciones hubo entre los tratamientos y los diagnósticos psiquiátricos. ${ }^{1}$

Para el abordaje de esta problemática se eligió el hospital J.A. Esteves de Lomas de Zamora -institución para mujeres que fue, hasta la década de 1940, un Anexo del Hospital Nacional de Alienadas (HNA), el hospital psiquiátrico para mujeres más importante del país-. Su carta fundacional revela haber sido un espacio creado para trasladar pacientes que no podían ocupar un lugar en el asilo de la capital, el HNA. El objetivo primero fue entonces trasladar a aquellas mujeres que se consideraban intratables, para liberar el espacio del hospital capitalino. De este modo fue creado sin pretensión de practicar terapéutica alguna, pero a raíz de ciertas coyunturas administrativas, más la acción de los médicos, se convirtió en una especie de banco de pruebas donde se llevaron a cabo los tratamientos que fueron llegando al país, desde la laborterapia hasta la aplicación de psicofármacos y psicoterapias. ${ }^{2}$

Se sostiene que las formulaciones teóricas y conceptuales sobre dichas terapias, así como el uso clínico de los tratamientos, son muestras de cómo era la mirada médica de la época; por lo tanto, es preciso estudiar ambos campos del trabajo psiquiátrico para conocer cómo los psiquiatras interpretaron la patología mental en el encuentro con sus pacientes. De este modo, se analizan dos escenarios bien diferenciados: el conformado por el ámbito académico, representado por estos artículos que circulaban por el "centro", y el del hospital, que conformaría la "periferia", encarnado por los expedientes médicos de las pacientes crónicas. Además, se hace hincapié en el trato de dichas terapias en cuadros de demencia precoz y de esquizofrenia, ya que el interés por éstos colonizó a la psiquiatría del siglo XX, tanto en su puesta en práctica en los ámbitos hospitalarios, como en las teorizaciones al respecto (a modo de ejemplo véase Novella y Huertas (2010).

\section{LAS TERAPIAS DE SHOCK, SUS PIONEROS Y SU ESTU- DIO COMO OBJETO HISTÓRICO}

Con este conjunto de terapias se buscó obtener mejorías en el cuadro mental del paciente a partir de la provocación de convulsiones, que podían derivar de la aplicación de una sustancia (insulina o cardiozol) o de una descarga eléctrica. A continuación, se formará un recorrido por el desarrollo de estas terapias, y se hará referencia tanto a los primeros en aplicarlas, como a su propagación en EEUU (principal polo de su desarrollo), y a algunas líneas de investigación trazadas al respecto.

Un rasgo que se repite en la literatura especializada sobre las terapias convulsivas es el valor que tuvieron como práctica para los psiquiatras. El clásico autor norteamericano Edward Shorter sostiene que, en la primera mitad del siglo XX en Estados Unidos, los psiquiatras se enfrentaban a un dilema: o dejaban a los pacientes en los hospicios a la espera de una cura espontánea o acudían al psicoanálisis, que no respondía a las necesidades de las enfermedades psiquiátricas. La posibilidad de recurrir a las terapias de shock desde la década de 1930 les permitió correrse del lugar de desesperanza en el que se encontraban, a pesar de las duras críticas que dichos tratamientos generaban (Shorter, 1997; Shorter y Healy, 2007). Además, 
representaron un hito para el campo de la psiquiatría, al romper el tutelaje de la neurología y sacar a la disciplina del lugar de "cenicienta" en los asilos. Así se subraya que se podía cuestionar si estas terapias hacían bien a los pacientes, pero con certeza fueron beneficiosas para la psiquiatría (Shorter, 1997, p. 224).

Deborah Doroshow, por su parte, retoma una de ellas en particular, el coma insulínico, y manifiesta que la mayoría de los historiadores de la psiquiatría entendieron su aplicación de dos modos: como un momento vergonzoso en el proceso de la psiquiatría biológica, o como un tratamiento somático más entre los usados para tratar la enfermedad mental a mediados del siglo XX, sin otorgarles demasiada relevancia. Para dicha autora, esta terapia supuso un momento clave en el desarrollo de la psiquiatría norteamericana, que les permitió a los psiquiatras, por un lado, acercarse al mainstream médico -y en especial a la neurología- $y$, por otro lado, encontrar una práctica eficaz. Desde la década de 1930 y durante veinte años, fue utilizada por todo Estados Unidos, y se confiaba en su eficacia clínica a pesar de la incertidumbre que se manifestaba en la literatura especializada. Su "capacidad curativa" para los casos de esquizofrenia les daba a médicos y enfermeras la confianza en su posibilidad de manipular el curso natural de una enfermedad de la que conocían muy poco. A pesar de sus riesgos, el coma insulínico permitió a los profesionales pensar que podían hacer algo por sus pacientes esquizofrénicos, lo cual los posicionaba como legítimos médicos (Doroshow, 2007, pp. 213-243). ${ }^{3}$

El personaje más destacado en la historia de la insulinoterapia fue el austríaco Manfred Sakel (19001957). Graduado en Medicina por la Universidad de Viena, debido al antisemitismo que se vivía en dicha ciudad, se exilió en Berlín en 1925, para trabajar en la clínica privada de Kurt Mendel, Lichterfelde Sanatorium. En 1933 retornó a Viena, y consiguió -bajo el tutelaje Otto Poetzl- un cargo en la clínica psiquiátrica de la universidad, donde empezó a probar el shock insulínico como cura de la esquizofrenia (Shorter, 1997, p. 209). Los primeros resultados de esta experiencia fueron expuestos en 1934 y, aunque Sakel sufrió el descrédito de sus colegas y la técnica no fue adoptada en Europa central, sí lo fue en el mundo anglosajón, particularmente en Suiza, bajo la figura de Max MüIler (Shorter, 1997, pp. 211-214). En 1936, Sakel viajó a Estados Unidos, donde otro médico, Joseph Wortis (1906-1995), ya había introducido esta terapia luego de observar el trabajo de aquel en Viena un par de años antes. Una vez en el nuevo continente, Sakel tra- bajó primero en el New York State Psychiatric Hospital y luego en una práctica privada en Manhattan. Para la década de 1960, había más de cien unidades de insulinoterapia en todo Estados Unidos (Shorter, 1997, p. 210). Más allá de este derrotero, Shorter considera que las convulsiones con insulinoterapia no habían sido buscadas, y que la convulsiterapia empezó realmente con el cardiozol -denominado Metrazol en Estados Unidos- introducido por Ladislao Von Meduna (Shorter, 1997, pp. 211-214). ${ }^{4}$

El médico húngaro Ladislao Von Meduna (18961964) se formó como neuropatólogo en el Interacademic Institute for Brain Research de Budapest. Luego siguió a su profesor Karl Schaffer al departamento de Psiquiatría de la Universidad de Budapest. Así como otros psiquiatras durante las décadas de 1920 y 1930, consideraba que existía un antagonismo entre la esquizofrenia y la epilepsia. Sostenía que, si los epilépticos estaban protegidos de la esquizofrenia, entonces inducir síntomas epilépticos podría servir de terapéutica para los esquizofrénicos. Desde esta presunción, desarrolló una terapia convulsiva a partir de un químico, el cardiozol, que fue calificada por los clínicos como peligrosa, por lo que poco a poco fue reemplazada por el electroshock, de la que fue su precursora. Con el electroshock se buscaba generar en los pacientes el mismo efecto convulsivo, pero con un riesgo menor. En 1939, von Meduna migró a Chicago, donde trabajó como profesor en Universidad Loyola en primer término, y luego en la Escuela de Medicina de la Universidad de Illinois (Shorter, 1997, p. 217).

Para 1938, mientras ya se aplicaban las otras terapias de shock en EEUU, el shock eléctrico aún estaba en proceso de desarrollo. Los italianos Ugo Cerletti (1877-1963) y Lucio Bini fueron los pioneros en la terapia de electroshock durante la década de 1930 . El primero había estudiado histología cerebral en HeideIberg, luego, hasta la Primera Guerra Mundial trabajó en la Clínica Psiquiátrica de Roma e hizo estudios de posgrado en diversos países, como Francia e Italia (Shorter, 1997, pp. 218-219). Lucio Bini trabajaba en la Universidad La Sapienza de Roma. Cerletti había estudiado con animales, a los que generaba convulsiones mediante electricidad, pero ninguno sobrevivió a sus estudios. Bini le sugirió entonces cambiar la ubicación de los electrodos, y así logró que los animales sobrevivieron a las siguientes pruebas. Para 1938 se hizo la primera prueba en humanos (en un hombre psicótico). Poco tiempo después, esta terapia se había propagado por todo Estados Unidos y algunos países de Europa (como Gran Bretaña, Alemania, Francia y 
España). No se la consideraba novedosa, sino que más bien era entendida como una mejora en el grupo de las convulsoterapias. Uno de los motivos por los que se popularizó fue porque, a pesar de haber sido pensada para tratar la esquizofrenia, al poco tiempo se la utilizó para diversos diagnósticos, como manía y depresión. Además, se creía que su utilización (como todas las terapias de choque) había permitido manejar mejor las instituciones hospitalarias (Tourney, 1967; Grob, 1991; Kingsley, 2000; Sadowsky, 2006; Doroshow, 2007, entre otros).

\section{TERAPIAS DE SHOCK EN EL ÁMBITO LOCAL: SUS TEORIZACIONES}

El tema de las terapias de shock fue incluido en las discusiones del campo de la psiquiatría argentina desde mediados de la década de 1930. Todos los artículos en revistas médicas y las tesis lo abordaban de manera similar: hacían una breve descripción de sus características, tiempo necesario de tratamiento y complicaciones que traía aparejadas para los pacientes; posibles combinaciones entre ellas y con la psicoterapia; indicaban las investigaciones que se realizaban a partir de su aplicación, los eventos científicos en los que eran objeto de debate y qué médicos e instituciones destacadas del ámbito local e internacional -principalmente europeos- las ponían en práctica (como ejemplos locales aparecían el Hospital de Alienados de Rosario y el HNA). Además, se indicaban los cuadros clínicos que presentaban los pacientes que las recibían, y se subrayaba su aplicación en casos de esquizofrenia. Para esta investigación se utilizaron principalmente tesis doctorales escritas para obtener el título de Doctor en Medicina, de la Universidad Nacional de Buenos Aires; también se encontraron artículos que se ocuparon de estas temáticas en boletines de diversos hospitales. El haber abordado el tema en tesis, implica que se trató de asuntos innovadores y en auge dentro de la disciplina. La publicación en boletines manifiesta la búsqueda de los psiquiatras en articular su trabajo clínico con el desarrollo teórico.

Todas las terapias que provocaban convulsiones aparecían caracterizadas en los textos como convulsoterapia. ${ }^{5}$ Luego, se hacía una distinción entre insulinoterapia, cardiozolterapia y electroshock. Se sostenía la necesidad de realizar psicoterapia (no se definía de qué tipo) en forma paralela a estos tratamientos ya que, a pesar del esfuerzo que implicaba para los médicos, se indicaba que eran notorias las diferencias entre pacientes que la recibían y los que no. Un artículo de 1939 sostenía que una vez que el cardiozol o la insulina hacían efecto, la psicoterapia podía reconstruir la personalidad del paciente para una mejor adaptación al medio ambiente. (Bula y Vita, 1939, p. 87-101).

A partir de la lectura de los textos científicos es claro que, en Argentina, primero se había pensado la terapia de electroshock sólo para la esquizofrenia (tomando como base las ideas del antagonismo entre esquizofrenia y epilepsia, que se describirá en profundidad en el apartado siguiente) y, debido a que los textos describían un supuesto éxito con esta patología, el tratamiento luego se extendió a las psicosis maníaco-depresivas, psicosis reactivas, episodios agudos de frenastenias, psicosis confusionales del climaterio, etc. (Bula y Vita, 1939, p.87-101). Para el caso de la insulinoterapia, se destacaba el uso en cuadros con psicosis melancólica, las formas ansiosas, estuporosas, crónicas, las formas delirantes hipocondríacas algunas psicopatías, y en el tratamiento de la esquizofrenia.

Las terapias de shock ponían en discusión la prognosis de la esquizofrenia por su curabilidad en un alto porcentaje de casos (Lertora, 1941, p. 29-34). A continuación, se cita un párrafo que manifiesta esta nueva situación clínica que se generaba con el uso de estos tratamientos y la postura que consideraban los psiquiatras que debían tomar al respecto:

"[...] la curabilidad de los dementes precoces amplía enormemente nuestro horizonte psiquiátricoterapéutico y al obligarnos al tratamiento de los casos en las primeras fases de su evolución nos debe llevar fuera del manicomio para poner rápidamente los enfermos en nuestras manos. En este terreno debemos romper preconceptos de nuestro ambiente social y aun del ambiente médico: es absolutamente falso que la locura sea incurable puesto que la revalidación social de un alto porcentaje de casos, pronta y debidamente asistidos, es un hecho innegable". (Lertora, 1941, p.34)

Claramente se pensaba que había una nueva posibilidad para los psiquiatras de tomar medidas terapéuticas ante el sufrimiento de los pacientes. De este modo se justificaba su uso, más allá de las complicaciones y los riesgos que significaban para los pacientes, porque se razonaba que era mejor correrlos o enfrentar estas consecuencias, antes que dejarlos sufrir y morir. Así se manifestaba, por ejemplo, en un artículo de la década de 1930, donde se señalaba que la esquizofrenia, más que una enfermedad, era un estado que acompañaba la vida del enfermo y que no era compatible con la convivencia social:

"El número de esquizofrénicos pues, que pueblan los establecimientos psiquiátricos es crecido y resulta 
mortificante para el médico la acción pasiva de ser testigo diario de una decadencia psíquica y también física, lenta aunque fatal, de un buen número de sus enfermos". (Foz, Vita, Ansaldi y Ordoñez, 1937, p. 40-76).

Se explicaba además que se trataba pacientes precoces y que albergaban "la peor de las locuras". Ante esta situación, no se podía pensar en esto como un tratamiento cruel, ya que peor era dejarlos sufrir su dolor moral y el peligro de que terminaran en suicidio (Bula y Vita, 1939, p.87-101).

En este recorrido se destaca que los trabajos científicos se basaban en las experiencias clínicas con terapias de shock en diversas instituciones hospitalarias nacionales, además de lo que la literatura científica internacional comentaba sobre su aplicación. También se evidencia que fueron entendidas como la posibilidad concreta para los psiquiatras de tratar los cuadros mentales con tratamientos específicos y una tecnología particular, que les permitía ubicarse como pares con otras disciplinas médicas. Asimismo, la urgencia de tratar los cuadros dentro del primer año del curso de la enfermedad -tal como se expresa en una de las citas transcriptas-obligaba a los médicos a intervenir por fuera de los hospitales y de la atención de pacientes crónicos.

Por último, se enfatiza cómo a partir de la aplicación de estas terapias cambió la concepción sobre el paciente esquizofrénico, ya que se pensó en la posibilidad de cura, o al menos en tratar sus síntomas y lograr una remisión momentánea. Desde lo manifestado en las producciones teóricas, el uso de estas terapias implicó un momento clave de cambio en la mirada médica: quizás la condena de muerte y aislamiento que implicaban ciertos cuadros mentales había quedado atrás. Como se desarrollará más adelante, para las pacientes del Esteves que se estudian en esta oportunidad-que murieron en la institución- esto no aplicó.

Las terapias de choque dejaron poco a poco de estar en el centro de la escena de la disciplina psiquiátrica, y ese lugar fue ocupado por los psicofármacos, cuya aparición fue una bisagra en los tratamientos psiquiátricos a nivel mundial. En Argentina se utilizaron desde finales de los años cincuenta, pero la producción teórica al respecto cobró relevancia desde los sesenta y, con el transcurso del tiempo se convirtieron en protagonistas indiscutibles en la terapéutica hospitalaria. Los artículos sobre la temática fueron escritos en muchas oportunidades por psiquiatras cercanos al psicoanálisis, en un momento en que éste y la psicología impregnaban cada vez más el campo de la salud mental. La historiografía sostiene que su llegada al país influyó en el uso de psicoterapias por parte de los psiquiatras, ya que permitían tratar el problema desde ángulos diferentes: la psicoterapia buscaba escuchar a los pacientes y los psicofármacos permitían generar las condiciones en ellos para poder ser escuchados (Plotkin, 2001, p. 125). A su vez, la combinación de tratamientos medicamentosos con psicoterapias eclécticas permitía un ejercicio profesional como lo hacían otros médicos. Pero esto también quitaba valor a las competencias de los psiquiatras, pues para la aplicación de esta terapia ya no se necesitaba la mirada especialista que se le adjudicaba al alienista de otros tiempos (Dagfal, 2009, p. 486).

Esta incorporación tuvo lugar dentro de un período de cambios en la disciplina psiquiátrica, y en las instituciones hospitalarias hubo una convivencia de viejos y nuevos paradigmas. La aparición de los psicofármacos era vista como una nueva revolución en la terapéutica psiquiátrica, ya que suponía la idea innovadora de que una sustancia química pudiera influir sobre un proceso psíquico (Caparrós, 1962, p.153). ${ }^{6}$ Así, los textos sobres estas temáticas daban fecha de inicio a la "era de la psicofarmacología" desde principios de la década de 1950 (Goldemberg, Barenblit y Sluzki, 1964, p. 126), y durante un período convivieron con las terapias de choque, tanto en el ámbito teórico como en el clínico.

\section{PSICOANÁLISIS Y TERAPIAS DE SHOCK: UN RASGO CLARAMENTE ARGENTINO}

A partir de la década de 1940, la mayor producción teórica acerca de las patologías de demencia precoz y esquizofrenia se ocupó de las terapias que se practicaban para tratarlas: las terapias de choque (cardiozol, insulinoterapia y shock eléctrico) y la psicoterapia psicoanalítica.

En Argentina, el psicoanálisis empezó a ser apropiado por el campo de la psiquiatría a partir de 1920-1930 como consecuencia, entre otras cosas, de la crisis general del positivismo (Plotkin, 1997, pp. 52-53), por lo que antes de la creación de la Asociación Psicoanalítica Argentina (APA) en 1942, ya poseía un impacto sobre los círculos médicos y culturales (Plotkin, 1997, p. 47). Para comentar los aportes psicoanalíticos a ciertas cuestiones referidas al tratamiento de la psicosis, se toma a Enrique Pichon Rivière, personaje destacado que participó de la fundación de la APA y que fue un referente en el campo psicoanalítico argentino.

Pichon Rivière (1907-1977) nació en Suiza y se estableció junto a su familia en el noreste argentino. Luego de afincarse ya de adulto en Buenos Aires se insertó 
en círculos bohemios, se interesó por el movimiento surrealista y realizó investigaciones sobre Isidore Ducasse, conde de Lautreámont. Al incorporarse a los círculos psicoanalíticos porteños ya era un psiquiatra reconocido y discípulo del director del Hospicio de las Mercedes (donde trabajaba y dictaba seminarios) (Plotkin, 2001, p. 45; Dagfal, 2015, pp. 25-26).

Hugo Vezzetti explica parte de la obra de Pichon Rivière hasta la década de 1950, sin adentrarse en su desarrollo teórico en los sesenta, ni en su teoría sobre psicología social. Se retoma su estudio, ya que allí se documentan los aportes de este médico suizo acerca de las psicosis. De acuerdo con Vezzetti, Pichon Rivière consideraba al psicoanálisis como una disciplina científica a partir de la cual se podía fundar una nueva psiquiatría (Vezzetti, 1996, p. 248). Además, indica que, para sus primeros trabajos sobre psicopatología psicoanalítica había partido de sus observaciones sobre la epilepsia. Sostenía que esta patología era un cuadro unificado, similar a una psicosis, que se separaba de la histeria por el grado de regresión y el punto de fijación en que el ataque epiléptico quedaba asociado a una situación de muerte. Vezzetti considera que, para el psiquiatra suizo, el epiléptico encarnaba la figura de lo primitivo, que no podía frenar lo instintivo. Esta concepción se acercaba a lo que para Cesare Lombroso fue el prototipo de delincuente por atavismo, y que para Sigmund Freud era la introyección de un superyó sádico que castigaba a un yo sometido. Retoma Hugo Vezzetti que, en cierto sentido, Pichon Riviére invirtió el planteo freudiano, pues no partió de la neurosis sino de la psicosis para desarrollar sus ideas de psicopatología (Vezzetti, 1996, pp. 255-256).

Del desarrollo teórico de Pichon Rivière sobre la epilepsia se abordan dos puntos. Por un lado, manifestó que fue a mediados de la década de 1940 cuando comenzó a mostrar su voluntad de construir una nosología de las psicosis dirigida a lo que llamó "enfermedad única". Consideraba que en las psicosis y neurosis había rechazo a la vida instintiva, pero que la diferencia entre las dos era cuantitativa. ${ }^{7}$ Toda psicosis, a su entender, se desarrollaba en dos tiempos, el primero de los cuales condicionaba la aparición de una perversión latente como mecanismo de defensa para evitar la castración o negarla. En el segundo tiempo se presentaba la psicosis propiamente dicha como tentativa de parte del yo para negar la perversión y para calmar, ligada al conflicto neurótico desencadenado entre dichas tendencias y el yo que se opone a su realización (Pichon, 1946, p.1-22). A partir de esta primera versión nosográfica surgieron la melancolía (con fantas- mas de aniquilamiento sobre el yo) y la esquizofrenia con la presencia de la disgregación.

Por otro lado, la epilepsia le generó al autor una matriz de tratamiento a partir del shock convulsivo. La explicación sobre la necesidad de provocar una convulsión epiléptica era que con ella se resolvería la tensión que existía entre el yo y el superyó, ya que de esta forma el paciente descargaría energías relacionadas con el instinto de muerte y la libido homosexual, de acuerdo con un mecanismo que el psicótico no epiléptico no podía realizar. Como conclusión, decía que era necesario provocar una epilepsia artificial en estos enfermos a partir del shock convulsivo. Vezzetti explica que para Pichon Rivière la terapéutica convulsivante no tenía una propiedad curativa, sino que hacía posible una operación sobre el yo (Vezzetti, 1996, pp. 258-259).

El médico suizo consideraba que el psicótico, a través del tratamiento de shock, modificaba el aspecto cuantitativo de sus tensiones instintivas, al descargar su agresividad por medio de la convulsión y el coma, denominados "mecanismos de muerte". Aclaraba que las condiciones psicodinámicas que habían motivado la esquizofrenia no se modificaban. Los contenidos patológicos solamente se reprimían al disminuir sus cargas, y permanecían más o menos inactivos en el inconsciente durante cierto tiempo hasta que hacían su nueva aparición. Sostenía que con la insulinoterapia en cierto momento se lograba que disminuyera la intensidad de las represiones y que se desbloquearan los contenidos reprimidos. Así el paciente pasaba a ser neurótico y a generar transferencia con el médico momentáneamente. El tratamiento debía comenzar con shock hipoglucémico y, en el caso de fracasar éste o de haberse reducido totalmente la sintomatología psicótica del enfermo, se debía recurrir al cardiozol o al electroshock (Pichon, 1946, p.1-22).

Por último, en relación con el procedimiento, Pichon Rivière sostenía que el médico que realizaba la psicoterapia no debía ser el mismo que aplicara los tratamientos biológicos, porque esto podía generar un aumento de ansiedad en el enfermo y provocar que se retirase a posiciones más lejanas a la realidad. Indicaba que el tratamiento psicoanalítico para la esquizofrenia difería del tratamiento con el neurótico en relación con la conducta del analista, pero no desde su dinámica. En ambos casos se giraba alrededor de la transferorresistencia. La psicoterapia en la esquizofrenia dependía del hecho de que el analista comprendiera la importancia de estos fenómenos de transferencia y de que lograra enfrentarlos de manera apropiada. 
Estos trabajos demuestran los cruces epistemológicos de la psiquiatría en busca de respuestas sobre la patología mental y el interés de diferentes ramas de la disciplina por los tratamientos para la psicosis. De este modo, más allá de la singular y atrapante figura de Pichon Rivière, su producción teórica acerca de las terapias de shock abre un nuevo escenario donde, por un lado, se describen nuevas hipótesis sobre la presencia de cuadros psicóticos, y por otro se justifica el uso de tratamientos de choque a partir de nociones psicoanalíticas, como un modo de propiciar las condiciones necesarias para practicar psicoterapia.

\section{EL ESTEVES COMO UN CASO CONCRETO DEL TRABA- JO CLÍNICO CON LAS TERAPIAS DE SHOCK}

En este artículo se trabaja en el ámbito clínico con una población muy particular: pacientes del Hospital José A. Esteves. Ellas eran, por lo general, de bajos recursos, la mitad eran extranjeras, muchas de las cuales no hablaban español, y en todos los casos presentaron al momento de su ingreso un cuadro psiquiátrico más o menos agudo que generó la preocupación de su pareja, un familiar o alguna institución pública, quien decidió internarla en un hospital psiquiátrico considerando que no se encontraba en condiciones de vivir una vida normal. Una vez internadas, estas mujeres sufrieron un proceso de cronificación hasta su muerte, y sólo un número acotado de ellas recibieron algún tratamiento, el cual no implicó la cura.

Las terapias de choque en el Esteves aparecieron dentro de un marco institucional que ya se había mostrado desde un principio como un lugar donde se utilizaban diversas herramientas y tratamientos de la psiquiatría en la primera mitad del siglo XX. Así, desde la apertura del hospital, se usó el trabajo entendido en el marco del tratamiento moral, y como un hecho natural para el desarrollo de las instituciones hospitalarias en el país. Además, el trabajo de las pacientes se destinaba específicamente al autoabastecimiento del hospital, pues no aparecen registros que permitan interpretar que su producción haya generado un ingreso económico para la institución, o para ellas mismas.

En el hospital Esteves, al igual que en otras instituciones públicas de Argentina, se mezcló el uso del trabajo como modo de mantener ocupadas a las pacientes, con la falta de personal para conservar principalmente la limpieza del lugar. Claramente la cuestión presupuestaria en una institución de estas características fue siempre un problema, y la participación de pacientes en el mantenimiento fue imprescindible. ${ }^{8}$
Luego, a partir de la década de 1930, hubo algunos (pocos) casos de práctica del tratamiento de piretoterapias. ${ }^{9}$ Éstos consistían en inocular diversas sustancias en enfermos mentales, con la idea de que los procesos infecciosos podían mejorar sus cuadros. Entre estas sustancias se utilizó la sangre de pacientes que padecían paludismo -malarioterapia-, un tratamiento considerado seguro y eficaz. Supuestamente, luego de las altas fiebres que producía la inoculación de parásitos de la enfermedad, los pacientes mejoraban. ${ }^{10}$ También fueron comunes el uso de ampollas de leche, restraint y chalecos de fuerza, como modos de contener a las mujeres, en especial previamente a la llegada de los psicofármacos.

Las historias clínicas de las pacientes que eran sometidas a los tratamientos de shock en el Hospital Esteves muestran mejoras en el corto plazo, pero en todos los casos se describe que los síntomas retornaron, aunque fuera de un modo más moderado; es decir, no manifestaron cambios profundos en los cuadros psiquiátricos. Por ejemplo, en la historia clínica de Teresa se registró, en junio de 1941:

\footnotetext{
"Terminó el tratamiento instituido con un total de 16 'shocks eléctricos' de los cuales 4 fueron frustros y los 12 restantes de mediana intensidad. Comenzó el electroshock con la dosis de 95 voltios durante 0,15 segundos y llegó a la dosis de 115 voltios durante 0,175 segundos". ${ }^{11}$

Cinco días después escribieron:

"Durante el tratamiento [...] se había esbozado una ligera mejoría, se alimentaba, solía levantarse [...] sueño normal. En la actualidad vuelve a retrogradar a su estado anterior. Hay que alimentarla [...] gritos, llantos, precisa hipnóticos para dormir". ${ }^{12}$
}

Esta última terapia se aplicaba, por lo general, con una frecuencia de día por medio durante un periodo de tiempo que variaba entre los 45 y los 90 días, con diferentes voltajes según la paciente. A algunas se les aplicaba en varias ocasiones a lo largo de su internación. Por su parte, los comas insulínicos aparecen de forma aislada en las historias clínicas. El uso del cardiozol se registraba en los expedientes médicos, pero no aparecen descripciones al respecto. Las terapias de choque eran aplicadas sin una explicación terapéutica concreta en las historias clínicas, y los tratamientos no eran excluyentes para algunos cuadros psiquiátricos, sino que se utilizaban para una gran variedad de diagnósticos e incluso para pacientes sin uno definido.

Estos tratamientos se combinaban, y en los expedientes médicos se registraban períodos de alternan- 
cia entre ellos. Ésta, por lo general, no muestra una justificación clínica o terapéutica, por lo que no es posible saber si se trataba de decisiones relativas al cuadro mental o basadas en cierta lectura teórica, o si respondían a la llegada de alguna tecnología nueva, o si el criterio se cambiaba por algún otro motivo.

Se pudieron detectar casos como el de Esther diagnosticada con esquizofrenia simple e ingresada en 1952-, en que el cambio de una terapia de shock a otra parece haber respondido a la falta de resultados de la primera. Así se describe que se le realizaron electroshocks cada tres días durante tres meses, y luego se aplicó insulinoterapia. En una nota se registró: "más comunicativa, se alimenta mejor [...]." Pero a continuación se manifestó: "mutismo absoluto, esteriotipia de movimientos, soliloquios [...] se indica insulinoterapia". Se siguieron describiendo síntomas como autismo y sonrisa estereotipada al entrevistarla y aparecen otros tratamientos de electroshock en los años 1957 y entre 1964 y 1966. ${ }^{13}$ Se sospecha que la repetición de los tratamientos puede haber respondido al uso de cada uno de ellos como un modo de aplacar momentáneamente ciertos síntomas, o que por coyunturas hospitalarias era el turno de una paciente para recibirlo. La realidad es que no se encuentran explicaciones clínicas en los expedientes médicos que justifiquen la aplicación de cada una de las terapias.

Continuando con esta última idea, en una historia clínica de la década de 1940, se presenta una planilla para el seguimiento del electroshock. En ella se buscaba registrar los siguientes datos: día de ingreso, nombre, edad, diagnóstico, fecha que se aplicaba la terapia, resistencia cefálica, voltaje, tiempo, características de la convulsión, accidentes, reacción posparoxismal, número de orden, modificaciones del cuadro clínico, y figura un espacio para registrar las observaciones. En este caso, se habían llenado los datos de fecha, voltaje, tiempo y número de orden. ${ }^{14}$ Se interpreta estos vacíos en las fichas como indicios de aquella información que en la práctica se consideraba relevante registrar para entender la aplicación terapéutica y la que no; aunque también pueden responder a falencias burocráticas del trabajo cotidiano en el hospital.

Con estas terapias se trataba el psiquismo de las pacientes, pero al mismo tiempo se hacía una intervención en su cuerpo. Todos los tratamientos implicaban una combinación de búsqueda de cura o mejoría de síntomas y una forma de control, ya que eran intervenciones que, dado su fuerte impacto físico, las calmaban (García, 1975). Es por esto que, a partir de la presencia de terapias de shock en el hospital, los médi- cos y el personal tuvieron nuevos mecanismos de contención de las mujeres que padecían algún ataque, y realizaron un control general del comportamiento de las pacientes en los pabellones. Se encontraron también expedientes médicos con descripciones sobre el uso de algunas de estas terapias combinadas con ampollas de leche o restraint para tranquilizarlas. ${ }^{15}$

Además, en algunos expedientes médicos figura el consentimiento de las familias para realizar cada una de las terapias. De hecho, aparecen casos de pacientes que se escapaban de la institución durante los tratamientos, y eran las mismas familias las que las llevaban de regreso al hospital. ${ }^{16}$ Esto presenta un escenario donde, si existía la presencia familiar, ellos conocían los tratamientos que las mujeres recibían.

Una referencia teórica obligada para entender el uso de los tratamientos en un escenario clínico como éste es la teoría de control social planteada como trasfondo en el trabajo de Irving Goffman, ${ }^{17}$ de acuerdo con la cual existe una normatividad implícita en la práctica, aunque no se encontrara objetivada, que se narraba a partir de diversas coyunturas del proceso de internación. Así, describe la normatividad generada al tratar a todos los internados del mismo modo, aplicando la "doctrina psiquiátrica" más allá de las diferencias personales que hubiera entre ellos. El autor describe también cómo a partir de las desfiguraciones en los cuerpos de los que llama internados -como consecuencia de las terapias de shock, por ejemplo-, se generaba la pérdida de seguridad personal. Además, los individuos participaban de diversos actos indignos en su cotidianeidad en la institución, que generaban consecuencias simbólicas incompatibles con la concepción de su propio yo (Goffman, 2001). ${ }^{18}$

Por otra parte, Pierre Bourdieu explica que la violencia simbólica es la coerción que se instituye a través de la adhesión, en que el dominado no puede evitar al dominante y, por lo tanto, a la dominación. Los instrumentos de conocimiento que tiene el dominado -que forman parte de la misma estructura de dominación incorporada- hacen aparecer a esta relación como natural. La violencia simbólica es una violencia ignorada, que, gracias a la adaptación inconsciente de las estructuras subjetivas y objetivas, hace posible la incorporación de creencias que parecen dotadas de legitimidad, de lo que está en el orden de las cosas (Chevallier, Chauviré y Consigle, 2011, pp. 176-177; Bourdieu, 1999, p. 190). Mediante el concepto de violencia simbólica busca hacer visible una forma imperceptible de violencia cotidiana. El autor considera que a dicho tipo de 
violencia es complejo resistirse, ya que está al mismo tiempo en todas partes y en ninguna (Bourdieu y Eagleton, 2000). El aporte de Bourdieu se toma para este caso particularmente en lo vinculado con el rasgo de invisibilidad de la violencia simbólica. A partir de esta noción, se considera que, en la cotidianeidad hospitalaria, las relaciones de poder que se generaron, y que regían las dinámicas dentro de la institución, funcionaban en un plano implícito e inconsciente. Bourdieu sostiene que, a partir de los mecanismos de violencia simbólica, la opresión es más eficaz y por lo tanto más brutal; al absorberse como el aire, como dice el autor, los dominados se adaptan a la situación. Su cuadro mental las posicionaba como "dominadas" frente a sus familiares, al personal hospitalario y a los médicos. Esto se reflejó en la pérdida de sus pertenencias, de su singularidad, de su autonomía, del control sobre su futuro, y en los cambios en su situación legal.

Estos mecanismos de dominación se pueden ubicar además en los cuerpos de las pacientes, los cuales se encontraban completamente en manos de los médicos y del personal de la institución (excepto momentos en los que reaccionaban e intentaban resistirse a algún tratamiento, cuando pretendían escaparse o producían algún acto de violencia física contra ellas mismas o un tercero). Las decisiones sobre su terapéutica -entre ellas, la exposición a terapias de choque- eran tomadas por los médicos, una situación en la cual por lo general las mujeres ni siquiera pensaban en la posibilidad de negarse. En el marco de esta estructura hospitalaria, la naturalización e invisibilidad de este proceso estaba dado por el "saber psiquiátrico" que dominaba la relación con las pacientes.

Por último, en el plano institucional, entre los documentos de la Sociedad de Beneficencia de la Capital Federal (institución a cargo del hospital hasta aquel momento), se encontró una nota del mes de enero de 1948 , donde se solicitaba a la administración del hospital la compra de cuatro aparatos de electroshock y se expresaba que, con una población de 3200 enfermas, la institución no contaba con ese "[...] imprescindible elemento para el tratamiento de enfermas mentales". Además, se manifestaba que en realidad se necesitaban 13 aparatos, uno por pabellón. Luego del estudio de tres marcas diferentes, se decidió comprar cuatro aparatos que fueron descriptos como

“Aparatos electro shock marca 'IDE', modelo D. IMP., construidos con material sólido, resistente a movimientos bruscos, a presión de golpes, para medir tensiones regulables desde 20 a 135 voltios corriente alternada y periódica de 50 ciclos [...]". ${ }^{19}$
De este modo, las terapias de choque parecen haber sido una práctica común del hospital, de la que se encuentran indicios en el registro de su aplicación, el consentimiento de las familias para realizarlas y en los documentos que refieren a la adquisición de la tecnología necesaria. De igual modo, en el hospital existieron muchas terapias, para pocas pacientes, ya que -como analizaremos a continuación- dentro del grupo de mujeres que estudiamos, hasta la década de 1960, la mayoría no recibió terapia alguna durante sus años de internación.

\section{ANÁLISIS CUANTITATIVO DE LOS TRATAMIENTOS EN PACIENTES CRÓNICAS: LAS TERAPIAS DE SHOCK Y SUS COMBINACIONES EN EL ESTEVES}

Con el objetivo de comprender la representatividad de la aplicación de dichas terapias en un espacio hospitalario y su combinación con otros tratamientos, se pretende realizar en este apartado un análisis cuantitativo de su aplicación en el Hospital Esteves. Se decidió considerar específicamente aquellos tratamientos que más se repiten en las historias clínicas, entre los que se encuentran la aplicación de terapias de shock (insulínico, cardiozólico y eléctrico), junto con psicofármacos y entrevistas psicológicas. ${ }^{20}$ En relación con la aplicación de estas terapias, como primer dato cuantitativo se observa que el $35 \%$ de los casos analizados recibieron alguna de éstas o la combinación de varias de ellas. La categoría que se denomina "combinadas" podría aplicarse en casi la totalidad de los casos, y consistió en el uso de terapias farmacológicas con algún otro tratamiento (véase cuadro 1 ).

Pero fue recién en la década de 1960 cuando el número de pacientes que recibieron terapias fue mayor que el de aquellas que no recibieron ninguna. Es decir que, desde la aparición de estos tratamientos hasta la última década del estudio, se presentó una doble realidad en el hospital: por un lado, una institución en la que se practicaban las terapias en boga a nivel mundial -a un número reducido de la población- y, por otro lado, una mayoría de pacientes crónicas que no recibían ningún procedimiento.

El tratamiento que más recibieron todos los cuadros de psicosis fue farmacológico; en segundo lugar, la combinación de diversas terapias; y en tercer lugar se aplicó shock (cuadro 1).

La psicoterapia y el examen psicológico fueron prácticamente nulos desde el aspecto cuantitativo, y figura en historias de pacientes ingresadas desde la década de $1960 .{ }^{22}$ El cuadro № 2 grafica las terapias recibidas 
Cuadro 1. Pacientes con "terapias combinadas": frecuencia y desglose ${ }^{21}$

\begin{tabular}{|l|l|l|l|l|}
\hline Casos & Psicoterapia & $\begin{array}{c}\text { Terapia de } \\
\text { shock }\end{array}$ & Terapia farmacológica & Examen psicológico \\
\hline 2 & & & & \\
\hline 1 & & & & \\
\hline 79 & & & & \\
\hline 1 & & & & \\
\hline 13 & & & & \\
\hline
\end{tabular}

Fuente: Elaboración propia a partir del Fondo Documental Historias Clínicas del Hospital Esteves, AGN Intermedio, con base en los casos analizados

Gráfico 1. Aplicación de terapia de shock según diagnóstico

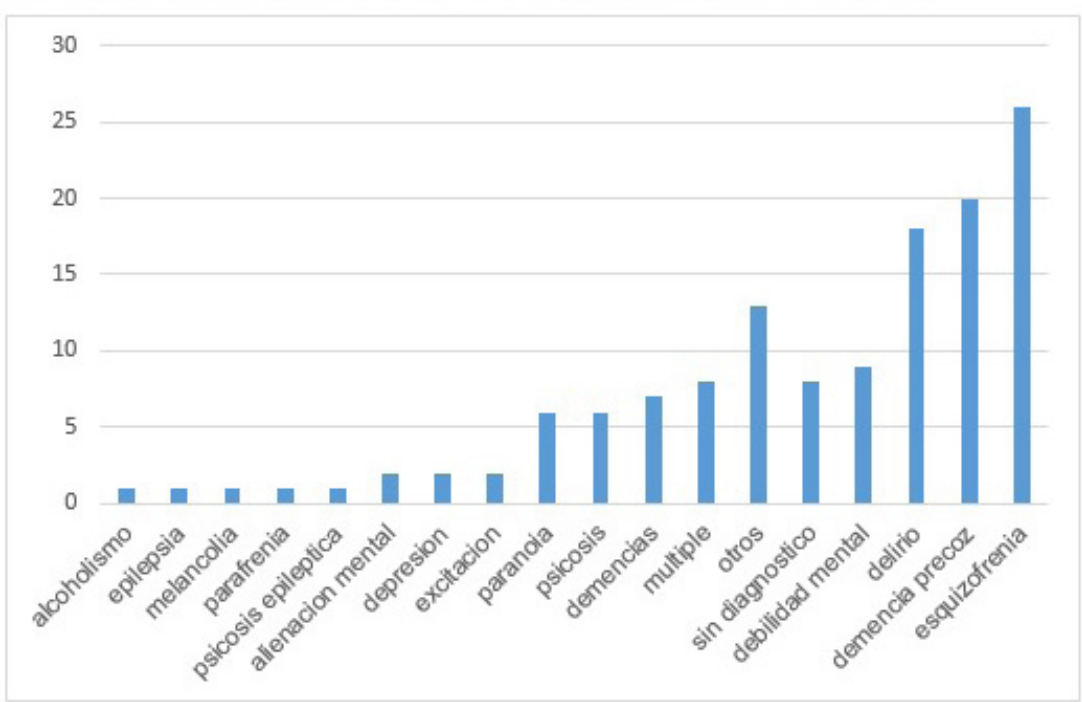

Fuente: Elaboración propia a partir del Fondo Documental Historias Clínicas del Hospital Esteves, AGN Intermedio, con base en los casos analizados), muestra el uso de la terapia de shock en todo el abanico diagnóstico.

según los distintos diagnósticos, que se definieron de la siguiente manera: primero se nombró "sin diagnóstico" a aquellos casos que no contaban con este dato; luego se separó las "no psicosis", es decir, todas las patologías psiquiátricas que no eran cuadros de psicosis. Entre éstos se hizo un corte cuantitativo y se destacaron los más representativos: demencia precoz, esquizofrenia, demencia, delirio, paranoia. Luego, a una cantidad importante de diagnósticos variados que no tenían entidad cuantitativa para ser considerados de manera particular se los agrupó con el nombre de "otras psicosis". Por último, se generó un grupo de "múltiple", que fueron aquellos casos que presentaron varios cuadros patológicos. El cuadro 2 destaca los tratamientos que recibieron las mujeres según su diagnóstico de psicosis.
El cuadro presenta dos extremos: las pacientes con delirio, que prácticamente no recibieron terapia, y las mujeres diagnosticadas con paranoia, que recibieron terapia casi en su totalidad. ${ }^{23}$ El principal motivo que explicaría los tratamientos de prácticamente todas las paranoicas fue que la paranoia fue la última de estas patologías en aparecer entre los diagnósticos del Esteves y, como ya se mencionó, cuanto más se avanza en el tiempo del arco temporal en estudio, más terapias recibieron las pacientes.

El segundo grupo que más terapias recibió fue el de "otras psicosis", más de la mitad de las que las recibieron, como muestra el cuadro 3. En este grupo se destacan las pacientes con cuadros que refieren a la epilepsia y diagnósticos vinculados a la senilidad. Por 
Cuadro 2. Aplicación de tratamiento según diagnósticos

\begin{tabular}{|l|c|c|c|}
\hline Diagnósticos & Cantidad de pacientes & $\begin{array}{c}\text { Pacientes que } \\
\text { recibieron terapia }\end{array}$ & Porcentaje \\
\hline Esquizofrenia y demencia precoz & 247 & 51 & $37 \%$ \\
\hline Demencia & 207 & 31 & $18 \%$ \\
\hline Delirio & 168 & 68 & $41 \%$ \\
\hline Múltiple* & 164 & 64 & $61 \%$ \\
\hline Otras psicosis & 105 & 14 & $82 \%$ \\
\hline Paranoia & 17 & 321 & $35 \%$ \\
\hline Totales & 907 & & \\
\hline
\end{tabular}

* El grupo "múltiple" está conformado por aquellos casos que presentaron varios cuadros patológicos, y que no se puede saber si estos diagnósticos les fueron adjudicados a las mujeres en una única admisión o si fueron variando a lo largo del tiempo.

Fuente: Elaboración propia a partir del Fondo Documental Historias Clínicas del Hospital Esteves, AGN Intermedio, con base en los casos analizados.

último, se puede notar que los cuadros clínicos que recibieron más cantidad de tratamientos fueron la demencia precoz y la esquizofrenia (si se trabaja con los totales), pues no se debe olvidar que también fueron los diagnósticos más numerosos (véase cuadro 3).

Las terapias que más se practicaron fueron las psicofarmacológicas, junto con las combinadas $Y$ las de shock como se muestra en el cuadro 4. A partir de su llegada en la década de 1950, los psicofármacos fueron utilizados en un mayor número de casos y poco a poco desplazaron a las terapias de shock. Se considera que este hecho puede responder principalmente a que su aplicación era más fácil y requería de menos personal que las terapias de choque, y también a su practicidad, pues a partir de las descripciones de algunos expedientes médicos se sabe que su uso permitió mantener bajo control a las mujeres en los pabellones. De este modo, la mayor eficacia de esta terapéutica implicó en primer lugar permitir el control de la población psiquiátrica, al modo de amansamiento por efectos químicos (véase cuadro 4).

Cuadro 3. Aplicación de tratamiento según diagnósticos en pacientes con "otras psicosis"

\begin{tabular}{|l|c|c|c|}
\hline & con terapia & sin terapia & Totales \\
\hline Diagnósticos que refieren a epilepsia & 14 & 9 & 23 \\
\hline Diagnósticos vinculados a senilidad & 35 & 18 & 29 \\
\hline Otros & 14 & 42 & 105 \\
\hline Totales & 63 & 15 & 53 \\
\hline
\end{tabular}

Fuente: Elaboración propia a partir del Fondo Documental Historias Clínicas del Hospital Esteves, AGN Intermedio, con base en los casos analizados.

En el caso del Hospital Esteves, los psicofármacos fueron utilizados en mayor proporción desde la década de 1960 y desde este momento el 30\% de las pacientes recibieron dicha terapia. No se encuentra una fundamentación sobre la decisión de medicar, o la elección entre el uso de una droga u otra, que más bien parece responder a la disponibilidad de los diversos psicofármacos. De igual modo, fueron casi nulos los casos en que haya existido un cambio en la medicación a lo largo de toda la internación. Por último, se destaca que 
Cuadro 4. Terapias practicadas en el Hospital Esteves ${ }^{24}$

\begin{tabular}{|l|c|c|}
\hline Terapias & Pacientes que las recibieron & Porcentaje \\
\hline Shock & 51 & $3,8 \%$ \\
\hline Psicofármacos & 296 & $22,2 \%$ \\
\hline Examen psicológico & 3 & $0,2 \%$ \\
\hline Psicoterapia & 1 & $0,1 \%$ \\
\hline Combinadas & 104 & $7,8 \%$ \\
\hline Sin terapia & 876 & $65,8 \%$ \\
\hline Total casos & 1331 & \\
\hline
\end{tabular}

Fuente: Elaboración propia a partir Fondo Documental Historias Clínicas del Hospital Esteves, AGN Intermedio, con base en los casos analizados.

los psicofármacos fueron utilizados para diversos cuadros diagnósticos y que el $11 \%$ de quienes recibieron esta terapia no tuvieron un diagnóstico registrado.

A partir del análisis realizado, se sostiene que el perfil del hospital para crónicas habría comenzado a cambiar con la llegada de terapias de choque, y por lo tanto el modo de entender los cuadros padecidos por estas mujeres también; pero al mismo tiempo seguía existiendo una mayoría de pacientes no tratadas que habitaban la institución y que murieron en ella. Es decir, la presencia de estas terapias fue un avance importante para los psiquiatras del Esteves, pues posicionó a este hospital a la par de otros nosocomios de la Capital Federal en materia de tratamientos, y probablemente (como se analizó en relación a la producción teórica de los psiquiatras) cambió la manera de entender a las mujeres, pero no implicó una modificación profunda en el tratamiento de pacientes crónicas.

\section{CONCLUSIONES}

El desarrollo teórico sobre las terapias de shock permite observar la presencia de las terapéuticas para la psicosis desde distintas posiciones teóricas. Además, la idea de cura era diferente según la postura teórica que se seguía: mientras que ésta era el utópico punto de llegada en las terapias biológicas, los psicoanalistas con sus tratamientos buscaban manejar síntomas. En el campo clínico, la idea unificada fue el uso combinado de las terapias de shock y farmacológicas con las psicoterapias: algo así como "paquetes" terapéuticos posibles de aplicar ante las patologías.

Al mismo tiempo, la llegada de estas terapias generó cambios en la concepción de los médicos sobre la enfermedad mental (al menos así lo manifestaron en sus textos), y ubicó a la esquizofrenia como un cuadro tratable -al menos en su etapa inicial-. Este cam- bio indefectiblemente debió modificar la imagen del enfermo mental, ya que su destino podía llegar a ser otro además de transitar el resto de su vida como paciente de una institución psiquiátrica.

A pesar de estos aportes teóricos, en los casos clínicos que se analizan, las terapias de choque parecen haber cumplido una función de respuesta ante la presencia de ciertos síntomas y momentos crisis de las pacientes, y no lograron modificar sus cuadros. Este contrapunto clínico no pretende desdecir aquello que se sostenía en la teoría, más bien busca mostrar otras realidades -las hospitalarias- que también sucedieron en el campo psiquiátrico local. Se destaca que las pacientes crónicas también fueron parte del escenario social, y que es preciso darles presencia como receptoras de estos tratamientos, por ejemplo, a partir de contar cómo fueron sus derroteros hospitalarios. Aunque la voz directa de las mujeres no aparezca en las descripciones realizadas de las historias clínicas, se considera que hay otros modos de darles visibilidad, por ejemplo, como se hizo en este trabajo, mediante el relato a partir de datos cuantitativos sobre tratamientos recibidos, el modo en que fueron diagnosticadas, y algunos rasgos personales (como su nacionalidad y el idioma que hablaban).

De este modo, se sostiene que los psiquiatras ensayaron modos de dar respuestas a las patologías mentales, particularmente a la esquizofrenia, a pesar de no entender demasiado sobre ella. Este hecho los ubica como personajes históricos que pretendieron paliar el sufrimiento de los enfermos, y que fueron construyéndose una identidad dentro de una disciplina particular inserta en el campo de la salud. Pero al mismo tiempo, es preciso destacar que estos tratamientos generaron dinámicas de poder particulares en el vínculo médico-paciente, y que tuvieron consecuencias físicas, psíquicas y emocionales en los pacientes. 


\section{AGRADECIMIENTOS}

Quiero agradecer la lectura y valiosos comentarios del Dr. Rafael Huertas, y de los evaluadores anónimos.

\section{NOTAS}

1 No todas las pacientes del Hospital Esteves fueron crónicas, pero para esta investigación se trabaja con aquellas mujeres que fallecieron en la institución.

2 Este hospital fue escenario de una de las experiencias psiquiátricas más innovadoras del país en la década del sesenta, el Proyecto Piloto de Comunidades Terapéuticas (19691971). Véase (Golcman, 2012).

3 Otros autores que abordaron estas terapias como objeto de estudio fueron (Scull, 1994) y (Braslow, 1997).

4 Véase (Berrios, 1997).

5 Acusse, Domingo (1945), "Cardiozolterapia en Psiquiatría", Tesis de Doctorado, Buenos Aires, Universidad de Buenos Aires, Facultad de Ciencias Médicas, Escuela de Medicina

6 Véase también Luna, J. A., Fernández Amallo, Cacliotti, C. y Bucich, G. (1969), "La trifluorperacina en la esquizofrenia. Su uso intratecal", Acta Psiquiátrica y Psicológica de América Latina, XV (1), pp. 91-95; Melgar, Ramón; Rodríguez Villegas, Raúl; Weledniger, Lola y Luna, Héctor Máximo (1960) “La trifluoperacina en el tratamiento de las esquizofrenias crónicas", Psiquiatría, 3. (1), p. 51-62; Goldemberg, Mauricio, Bar, Liliana, Caparrós, Antonio, Casarino, Marcelo, Germano, Carlos, Hammond, Harold, Kesselman, Hernán y Sluzki, Carlos (1961), "Nuestra experiencia en psicofarmacología clínica. Segundo Congreso Argentino de Psiquiatría". Tema II: Psicofarmacología clínica, Acta Neuropsiquiátrica Argentina, 7, pp. 83-84; Fischer, Edmundo (1961a), “Algunos Aspectos Psicofisiológicos de la Psicofarmacología”, Psiquiatría, 1 (4), pp. 61-66; (1961b), "Algunos enfoques clínicos de la psicofarmacología”, Psiquiatría, 3 (1), p. 67.

7 Pichon Rivière, Enrique (1946), “Contribución a la teoría psicoanalítica de la esquizofrenia", Revista de Psicoanálisis, 4 (1), pp. 1-22.

8 Entrevistas realizadas por la autora a L. V. y A. M., psicóloga y enfermera de la institución respectivamente, 19 de mayo de 2013.

9 En su origen, la malarioterapia fue propuesta por el médico austríaco Julius von Wagner-Jauregg (1857-1940). Su primer uso fue en 1917 para tratar la parálisis general progresiva. Véase (Brown, 2000) y (Villasante, 2003).

10 Desde 1918 se lo utilizó también para la demencia precoz. Véase (Conseglieri, 2008).
11 Historia clínica 5052, Legajo 21. Archivo General de la Nación, Departamento Intermedio. Hospital Neuropsiquiátrico Esteves de Lomas de Zamora. Para otros casos con descripciones similares véase Historia clínica 8574, Legajo 8. Archivo General de la Nación, Departamento Intermedio. Hospital Neuropsiquiátrico Esteves de Lomas de Zamora.

12 Historia clínica 5052, Legajo 21. Archivo General de la Nación, Departamento Intermedio. Hospital Neuropsiquiátrico Esteves de Lomas de Zamora. Para otros casos con descripciones similares véase Historia clínica 8574, Legajo 8. Archivo General de la Nación, Departamento Intermedio. Hospital Neuropsiquiátrico Esteves de Lomas de Zamora.

13 Historia clínica 8574, Legajo 8. Archivo General de la Nación, Departamento Intermedio. Hospital Neuropsiquiátrico Esteves de Lomas de Zamora.

14 Historia clínica 37.752, Legajo 9. Archivo General de la Nación, Departamento Intermedio. Hospital Neuropsiquiátrico Esteves de Lomas de Zamora.

15 A modo de ejemplo véase Historia clínica 39.944, Legajo 46. Archivo General de la Nación, Departamento Intermedio. Hospital Neuropsiquiátrico Esteves de Lomas de Zamora. Las ampollas de leche eran inyecciones que se les aplicaban generalmente en las piernas. Estas ampollas generaban fiebre, edemas e incapacidad para moverse, lo que las obligaba a mantenerse en reposo.

16 Historia clínica 8081, Legajo 11; Historia clínica 50.680, Legajo 25. Archivo General de la Nación, Departamento Intermedio. Hospital Neuropsiquiátrico Esteves de Lomas de Zamora.

17 Erving Goffman desarrolla su teoría sobre las instituciones totales, a las que explica como lugares de residencia y de trabajo donde gran número de individuos en igual situación, aislados de la sociedad por un período apreciable de tiempo, comparten en su encierro una rutina diaria, administrada formalmente. Su desarrollo hace especial hincapié en los internos de los hospitales psiquiátricos, y se propone exponer una versión sociológica de la estructura del yo, en una normatividad que estaba implícita en la práctica, aunque no se encontrara objetivada, y en una relación en la que uno mandaba y el otro obedecía, ya que la voz del psiquiatra era indiscutible. Véase (Goffman, 2001).

18 Otros trabajos clásicos que fueron contemporáneos a éste fueron (Szasz, 1961) y (Foucault, 2005). Véase también (Leonardis, 1992), (Huertas, 2009). 
19 Sociedad de Beneficencia, Expediente 29.332 Legajo 196. Actas de la Sociedad de Beneficencia de la Capital Federal. Archivo General de la Nación.

20 El período completo del análisis cuantitativo de pacientes crónicas fue entre 1895 y 1987, es decir, el arco temporal completo de las fuentes que contiene el archivo de Historias Clínicas de dicho Hospital en el Archivo General de la Nación, Departamento Intermedio. Sin embargo, como la aparición de las terapias de choque no fue hasta la década de 1930 en el hospital, este trabajo acota su periodización entre 1930 y la primera mitad de la década de 1970, momento en que se encuentra el último caso que recibió este tipo de terapéutica. El total de historias clínicas del acervo documental es de 4058. Se trabajó con alrededor de la tercera parte de los casos.

21 En el análisis cuantitativo de las pacientes que recibieron terapias no se cuenta con la fecha exacta de su aplicación, ya

\section{BIBLIOGRAFÍA}

Acusse, Domingo (1945), Cardiozolterapia en Psiquiatría, Tesis de Doctorado, Buenos Aires, Universidad de Buenos Aires, Facultad de Ciencias Médicas, Escuela de Medicina.

Berrios, Germán (1997), "The scientific origins of electroconvulsive therapy: a conceptual history", History of Psyquiatry, 8, pp. 105-120.

Bourdieu, Pierre (1999), Meditaciones pascalianas, Barcelona, Anagrama, p. 190.

Bourdieu, Pierre y Eagleton, Terry (2000), "'Doxa y vida ordinaria'. Pensamiento crítico contra la dominación", New Left Review, 0, pp.219-231.

Braslow, Joel T. (1997), Mental ills and bodily cures: psychiatric treatment in the first half of the twentieth century, California, University of California Press.

Brown, Edward M. (2000), "Why Wagner-Jauregg won the Nobel Prize for discovering malaria therapy for General Paresis of the insane?", History of Psychiatry, 11 (4), pp. 371-382.

Bula, Nélida y Vita, Nicolás (1939), "Psicosis melancólica y convulsoterapia", Boletín del Instituto Psiquiátrico, 3 (21), pp. 87-101.

Caparrós, Antonio (1962), "Bases para el uso de psicofármacos", Anales Argentinos de Medicina, VII (VII), p. 153.

Conseglieri, Ana (2008), "La introducción de nuevas medidas terapéuticas: entre la laborterapia y el electroshock en el manicomio de Santa Isabel", Frenia, 8 (1), pp. 131-160, p. 145. que no en todas las historias clínicas estaba registrada; por lo que se trata de entender esta periodización en el cruce entre la fecha de admisión de las pacientes y las décadas en las cuales surgieron estas terapias. Para los casos que se desarrollan en profundidad, están contempladas las fechas exactas de aplicación de los tratamientos.

22 No se debe perder de vista que la población que se analiza es sólo de aquellas pacientes que fallecieron en la institución, y este puede ser uno de los motivos posibles para entender la falta de casos que hayan recibido psicoterapias.

23 No se debe olvidar que dichos casos representan sólo el 1\% de las psicosis.

24 Si se suman las pacientes que recibieron terapia de shock como única terapia, con los casos que se aplicaron shock combinada con otras (132 casos), se destaca que esta terapia fue la más utilizada luego de los psicofármacos.
Chevallier, Stéphane; Chauviré, Christiane y Consigle, Estela (2011), Diccionario Bourdieu, Buenos Aires, Nueva Visión, pp. 176-177.

Dagfal, Alejandro (2009), Entre París y Buenos Aires. La invención del psicólogo (1942-1966), Buenos Aires, Paidós, p. 486.

Dagfal, Alejandro (2015), "El pasaje de la higiene mental a la salud mental en la Argentina, 1920-1960. El caso de Enrique Pichon-Rivière", Trashumante. Revista Americana de Historia Social, 5, pp. 10-36, pp. 25-26.

Doroshow, Deborah Blythe (2007), "Performing a Cure for Schizophrenia: Insulin Coma Therapy on the Wards", Journal of the History of Medicine and Allied Sciences, 62 (2), pp. 213-243.

Fischer, Edmundo (1961a), "Algunos Aspectos Psicofisiológicos de la Psicofarmacología", Psiquiatría, 1 (4), pp. 61-66.

Fischer, Edmundo (1961b), “Algunos enfoques clínicos de la psicofarmacología”, Psiquiatría, 3 (1), p. 67.

Foucault, Michel, (2005), El poder psiquiátrico, Madrid, Akal.

Foz, Antonio, Vita, Nicolás., Ansaldi, Inocencio, Ordoñez, José. (1937), "Insulinoterapia en psiquiatría", Boletín del Instituto Psiquiátrico, 1 (20-21), pp. 40-76.

García, Ramón (coord.) (1975), Psiquiatría, antipsiquiatría y orden manicomial, Barcelona, Barral.

Golcman, Alejandra (2012), "The experiment of the therapeutic communities in Argentina: the case of the Hospital Esteves", Psychoanalysis and History, 4 (2), pp. 269-284. 
Goldemberg, Mauricio, Bar, Liliana, Caparrós, Antonio, Casarino, Marcelo, Germano, Carlos, Hammond, Harold, Kesselman, Hernán y Sluzki, Carlos (1961), “Nuestra experiencia en psicofarmacología clínica. Segundo Congreso Argentino de Psiquiatría". Tema II: Psicofarmacología clínica, Acta Neuropsiquiátrica Argentina, 7, pp. 83-84.

Goldemberg, Mauricio, Barenblit, Valentín y Sluzki, Carlos (1964), "Los psicofármacos en psiquiatría", La Semana Médica, tomo 125 , I (28).

Goffman, Erving (2001), Internados. Ensayos sobre la situación de los enfermos mentales, Buenos Aires, Amorrortu.

Grob, Gerald (1991), From Asylum to Community: Mental Health Policy in Modern America, Princeton, Princeton University Press, p. 127

Huertas, Rafael (2009), "El nacimiento del manicomio. De la medicalización de la locura a la gestión de los 'residuos", Bostezo, 1 (3), pp. 42-45.

Kingsley, Jones (2000), "Insulin Coma Therapy in Schizophrenia", Journal of the Royal Society of Medicine, 93, pp. 147-149.

Leonardis, Ota de (1992), "Políticas sociales: reinventar nuevos parámetros". En: Álvarez-Uría, Fernando, y Bailleau, Francis (coords.), Marginación e inserción. Los nuevos retos de las políticas sociales, Madrid, Endimión, pp. 55-68.

Lertora, Adolfo (1941), "Shockterapia de las psicosis", Boletín del Hospital Melchor Romero, 1 (2), pp. 29-34.

Luna, J. A.; Fernández Amallo, J.; Cacliotti, C. y Bucich, G. (1969), "La trifluorperacina en la esquizofrenia. Su uso intratecal", Acta Psiquiátrica y Psicológica de América Latina, XV (1), pp. 91-95.

Novella, Eric J. y Huertas, Rafael (2010), “El síndrome de Kraepelin-Bleuler-Schneider y la conciencia moderna: una aproximación a la historia de la esquizofrenia", Clínica y Salud, 21 (3), pp. 205-219, pp. 212-215.

Melgar, Ramón; Rodríguez Villegas, Raúl; Weledniger, Lola y Luna, Héctor Máximo (1960) "La trifluoperacina en el tratamiento de las esquizofrenias crónicas", Psiquiatría, 3. (1), p. 51-62.
Pichon Rivière, Enrique (1946), "Contribución a la teoría psicoanalítica de la esquizofrenia", Revista de Psicoanálisis, 4 (1), pp. 1-22.

Plotkin, Mariano Ben (1997), “Freud, Politics, and the Porteños: The Reception of Psychoanalysis in Buenos Aires (19101943)", Hispanic American Historical Review, 77 (1), pp. 4574, pp. 52-53.

Plotkin, Mariano Ben (2001), Freud in the Pampas. The Emergence and Development of a Psychoanalytic Culture in Argentina, Stanford University Press, California, p. 125.

Sadowsky, Jonathan (2006), "Beyond the Metaphor of the Pendulum: Electroconvulsive Therapy, Psychoanalysis, and the Styles of American Psychiatry", Journal of the History of Medicine and Allied Sciences, 61 (1), pp. 1-25, https://doi. org/10.1093/jhmas/jrj001.

Scull, Andrew (1994), "Somatic Treatments and the Historiography of Psychiatry", History of Psychiatry, 5, pp. 1-12.

Shorter, Edward (1997), A History of Psychiatry. From the Era of the Asylum to the Age of Prozac, Nueva York, John Wiley.

Shorter, Edward y Healy, David (2007), Shock Therapy: A History of Electroconvulsive Treatment in Mental Illness, New Brunswick, Rutgers University.

Szasz, Thomas (1961), The Myth of Mental IIInes: Foundations of a Theory of Personal Conduct, Nueva York, Hoeber-Harper.

Tourney, Garfield (1967), "A History of Therapeutic Fashions in Psychiatry, 1800-1966", American Journal of Psychiatry, 124, pp. 784-796.

Vezzetti, Hugo (1996), Aventuras de Freud en el país de los argentinos. De José Ingenieros a Enrique Pichon Rivière, Buenos Aires, Paidós.

Villasante, Olga (2003), "La malarioterapia en el tratamiento de la Parálisis General Progresiva: Primeras experiencias en España". En: Fuentenebro, Filiberto; Huertas, Rafael y Valiente, Carmen (eds.), Historia de la psiquiatría en Europa. Temas y tendencias, Madrid, Frenia, pp. 175-188. 